Supporting Information for OM2019-002508

\title{
Syntheses and Structures of Triple-Decker Complexes Incorporating
}

\section{Octamethylruthenocene and Nonamethylruthenocene}

Joseph J. McBride, Timothy J. Casad, Emina E. Cheung, Robert D. Pike and Eric J. Watson*

Corresponding author: Eric J. Watson.email: watsone1@seattleu.edu

\section{Table of Contents}

S2. Table S1. Table of Crystallographic and structure refinement data for compounds $\mathbf{1} \cdot \mathrm{PF}_{6}$, $2 \cdot \mathrm{PF}_{6}, 3 \cdot \mathrm{PF}_{6}, 4 \cdot \mathrm{PF}_{6}$ and $\mathbf{5} \cdot \mathrm{PF}_{6}$.

S3. Figure $\mathrm{S} 1 .{ }^{1} \mathrm{H},{ }^{13} \mathrm{C}\{\mathrm{H}\} \mathrm{NMR}$ spectra for compound $\mathbf{1} \cdot \mathrm{PF}_{6}$.

S4. Figure S2. HMQC NMR spectrum for compound 1·PF6.

S5. Figure S3. ${ }^{1} \mathrm{H},{ }^{13} \mathrm{C}\{\mathrm{H}\} \mathrm{NMR}$ spectra for compound $2 \cdot \mathrm{PF}_{6}$.

S6. Figure S4. ${ }^{1} \mathrm{H},{ }^{13} \mathrm{C}\{\mathrm{H}\} \mathrm{NMR}$ spectra for compound $3 \cdot \mathrm{PF}_{6}$.

S7. Figure S5. ${ }^{1} \mathrm{H},{ }^{13} \mathrm{C}\{\mathrm{H}\} \mathrm{NMR}$ spectra for $\mathrm{Cp}^{\prime} \mathrm{RuCp}, \mathrm{CpRuCp}$ ' and CpRuCp.

S8. Figure S6. S4 ${ }^{1} \mathrm{H},{ }^{13} \mathrm{C}\{\mathrm{H}\} \mathrm{NMR}$ spectra for compound $4 \cdot \mathrm{PF}_{6}$.

S9. Figure S7. S4 ${ }^{1} \mathrm{H},{ }^{13} \mathrm{C}\{\mathrm{H}\} \mathrm{NMR}$ spectra for compound $\mathbf{5} \cdot \mathrm{PF}_{6}$.

S10. Figure S8. ${ }^{1} \mathrm{H},{ }^{13} \mathrm{C}\{\mathrm{H}\}$ NMR spectra for compound 6. 


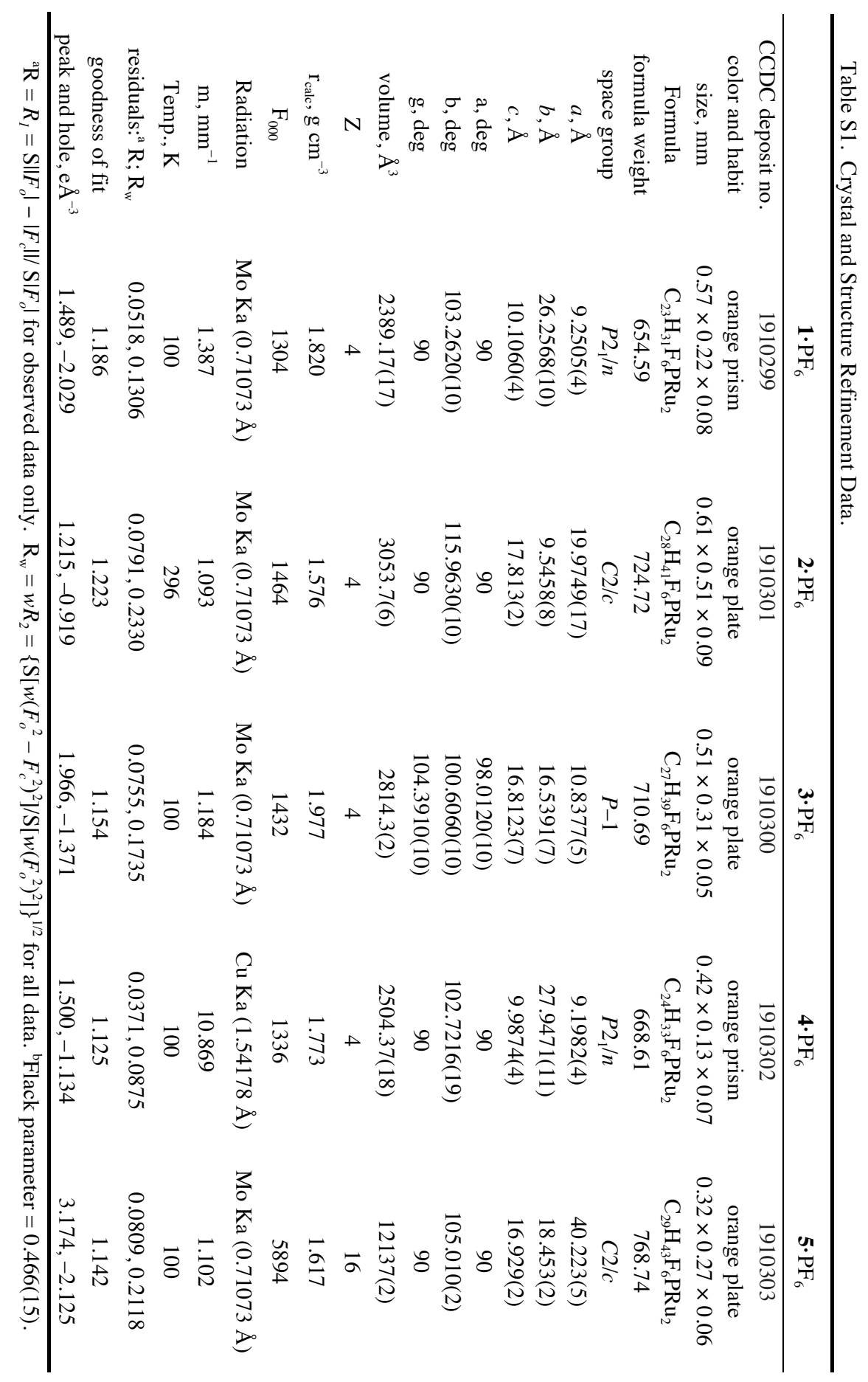


Figure $\mathrm{S} 1 .{ }^{1} \mathrm{H},{ }^{13} \mathrm{C}\{\mathrm{H}\} \mathrm{NMR}$ spectra for $\left[\mathrm{CpRu}\left(\mu-\mathrm{Cp}^{\prime}\right) \mathrm{RuCp}^{\prime}\right] \mathrm{PF}_{6}\left(\mathbf{1} \cdot \mathrm{PF}_{6}\right)$.
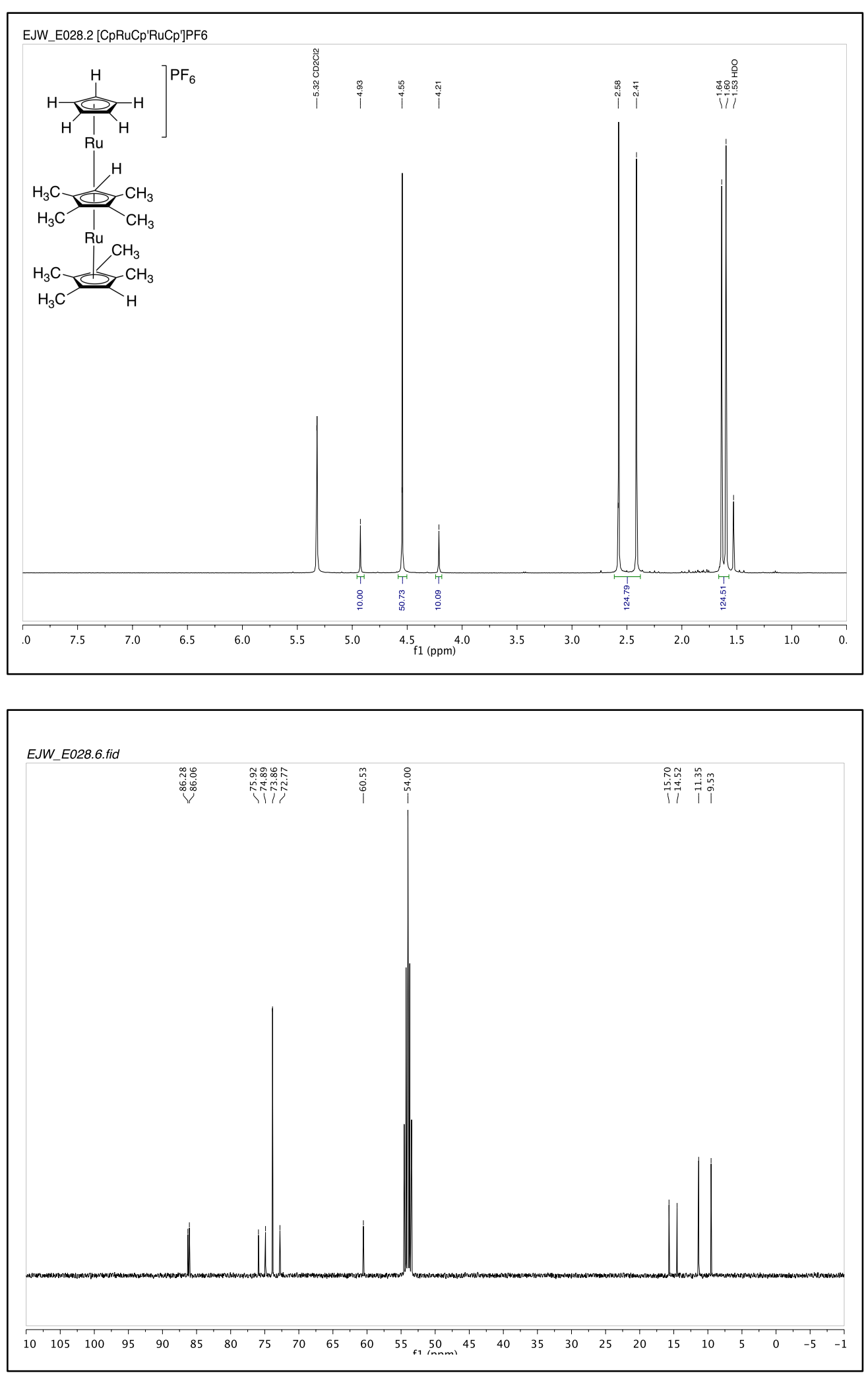
Figure S2. HMQC NMR spectrum for compound 1·PF6.

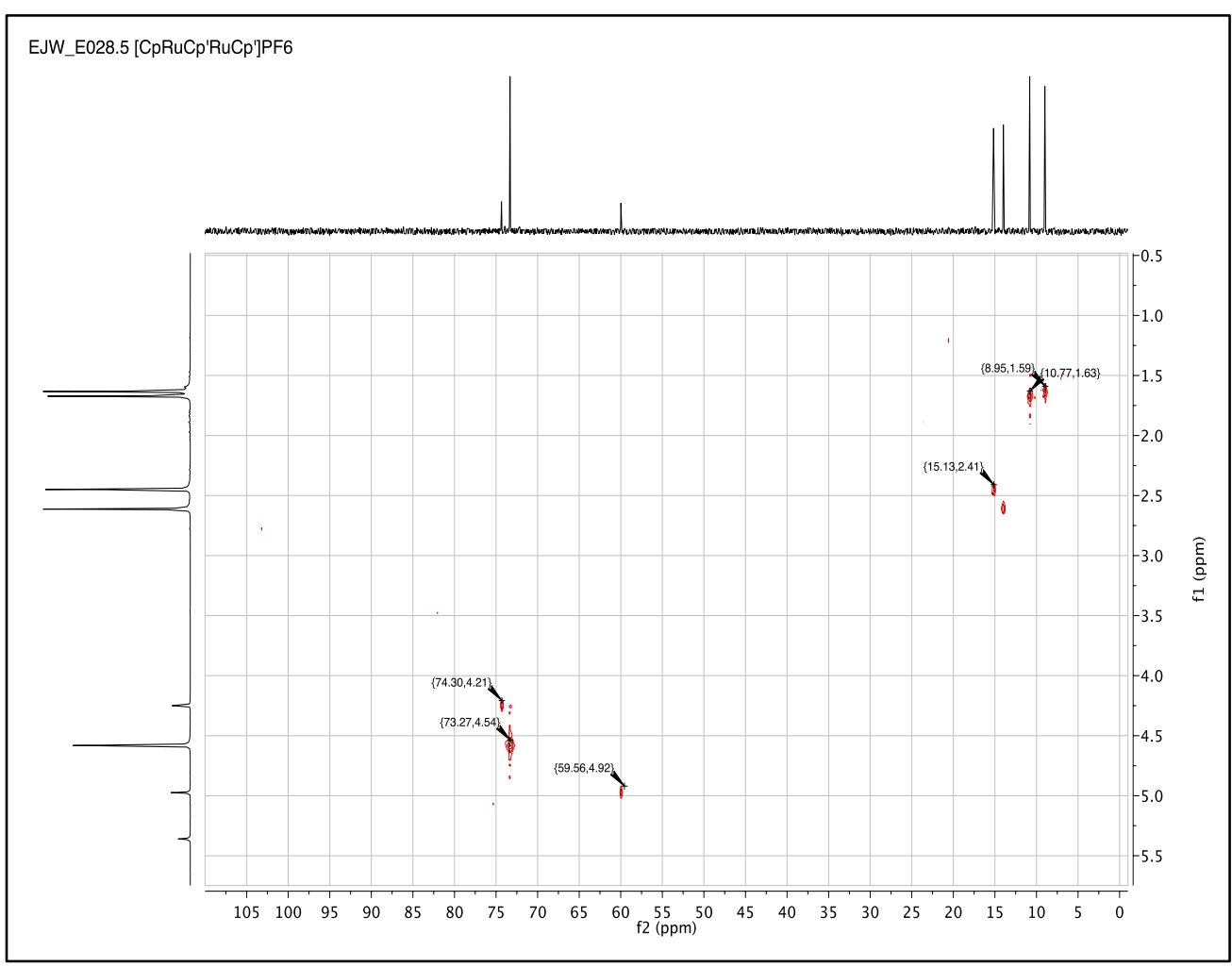


Figure S3. ${ }^{1} \mathrm{H},{ }^{13} \mathrm{C}\{\mathrm{H}\}$ NMR spectra for $\left[\mathrm{Cp} * \mathrm{Ru}\left(\mu-\mathrm{Cp}^{\prime}\right) \mathrm{RuCp}^{\prime}\right] \mathrm{PF}_{6}\left(\mathbf{2} \cdot \mathrm{PF}_{6}\right)$.
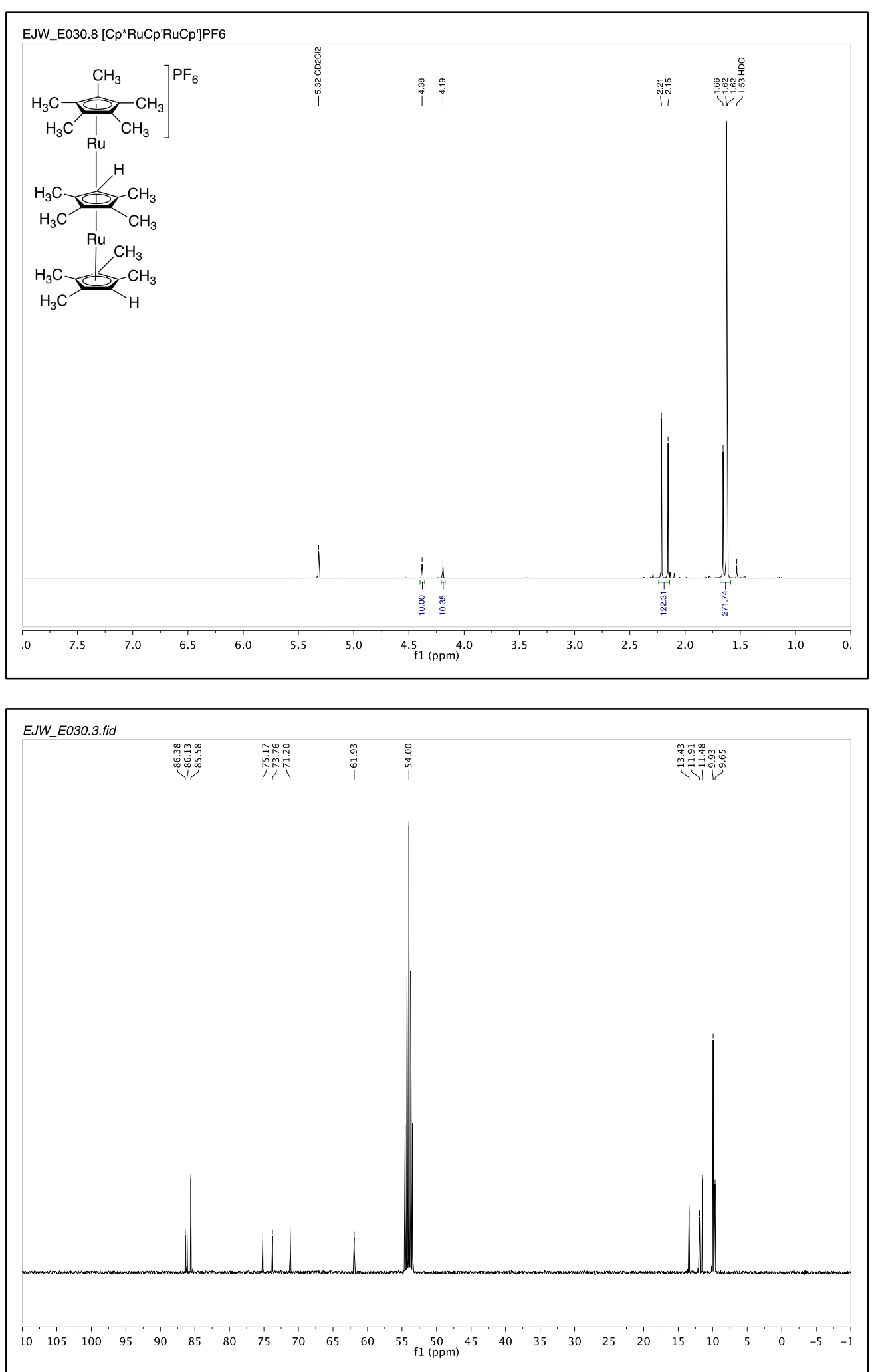
Figure S4. ${ }^{1} \mathrm{H},{ }^{13} \mathrm{C}\{\mathrm{H}\}$ NMR spectra for $\left[\mathrm{Cp} ' \mathrm{Ru}\left(\mu-\mathrm{Cp}^{\prime}\right) \mathrm{RuCp} \mathrm{CPF}_{6}\left(3 \cdot \mathrm{PF}_{6}\right)\right.$.
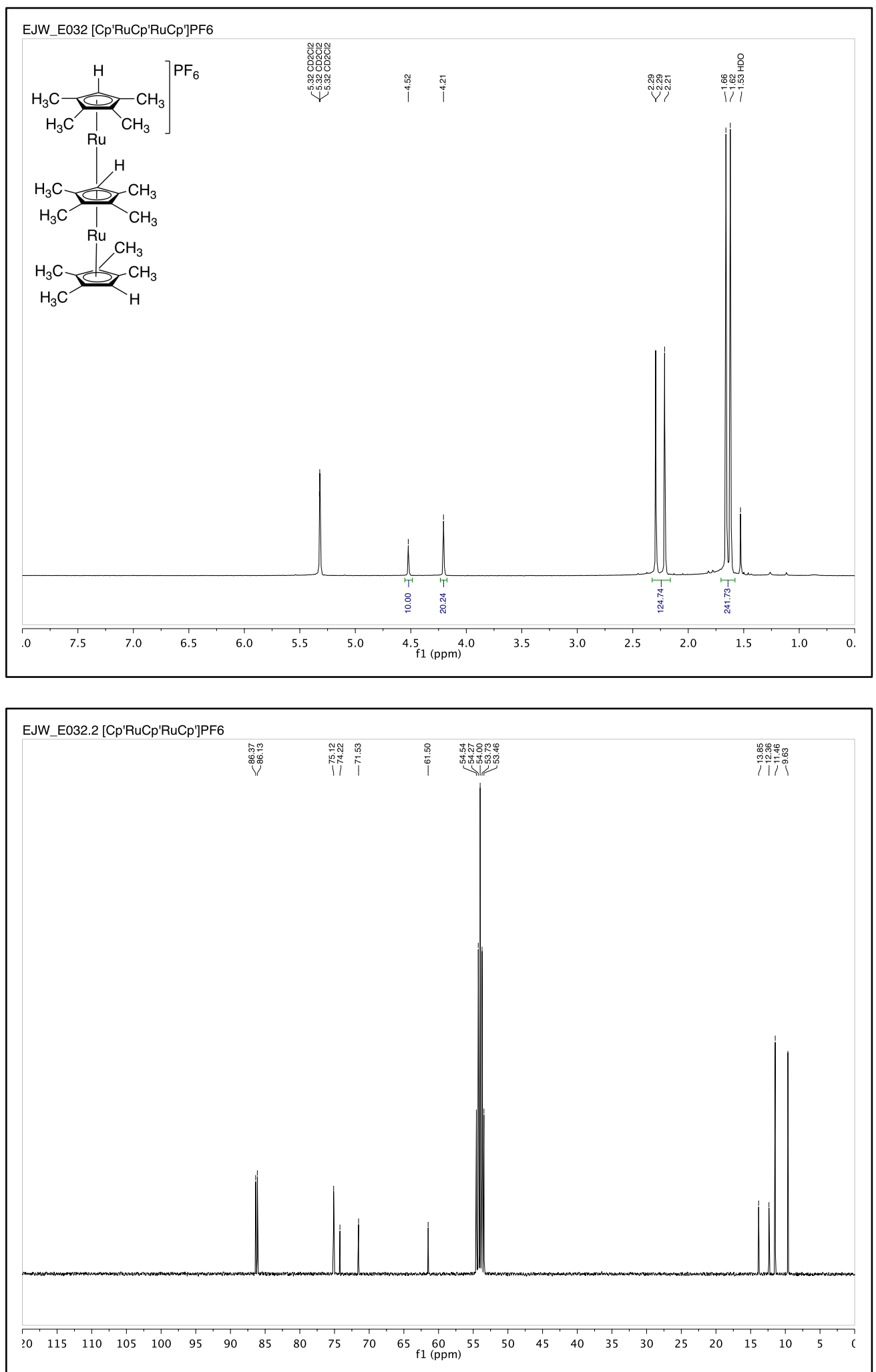
Figure S5. ${ }^{1} \mathrm{H},{ }^{13} \mathrm{C}\{\mathrm{H}\}$ NMR spectra for $\mathrm{Cp}^{\prime} \mathrm{RuCp}$ ', $\mathrm{CpRuCp}$ ' and $\mathrm{CpRuCp}$ in $\mathrm{CDCl}_{3}$.
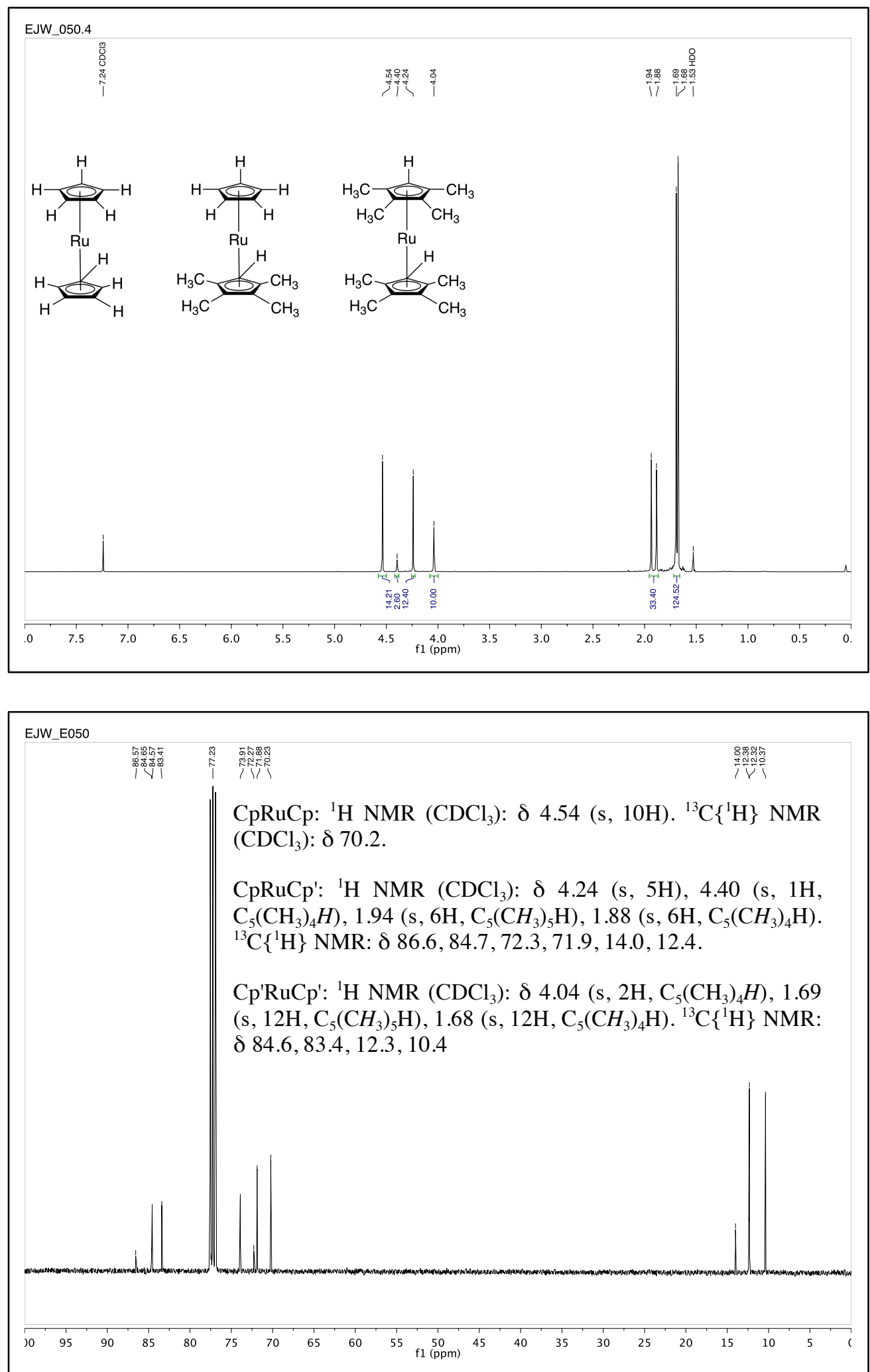
Figure S6. ${ }^{1} \mathrm{H},{ }^{13} \mathrm{C}\{\mathrm{H}\} \mathrm{NMR}$ spectra for $\left[\mathrm{CpRu}\left(\mu-\mathrm{Cp}^{\prime}\right) \mathrm{RuCp}\right] \mathrm{PF}_{6}\left(4 \cdot \mathrm{PF}_{6}\right)$.
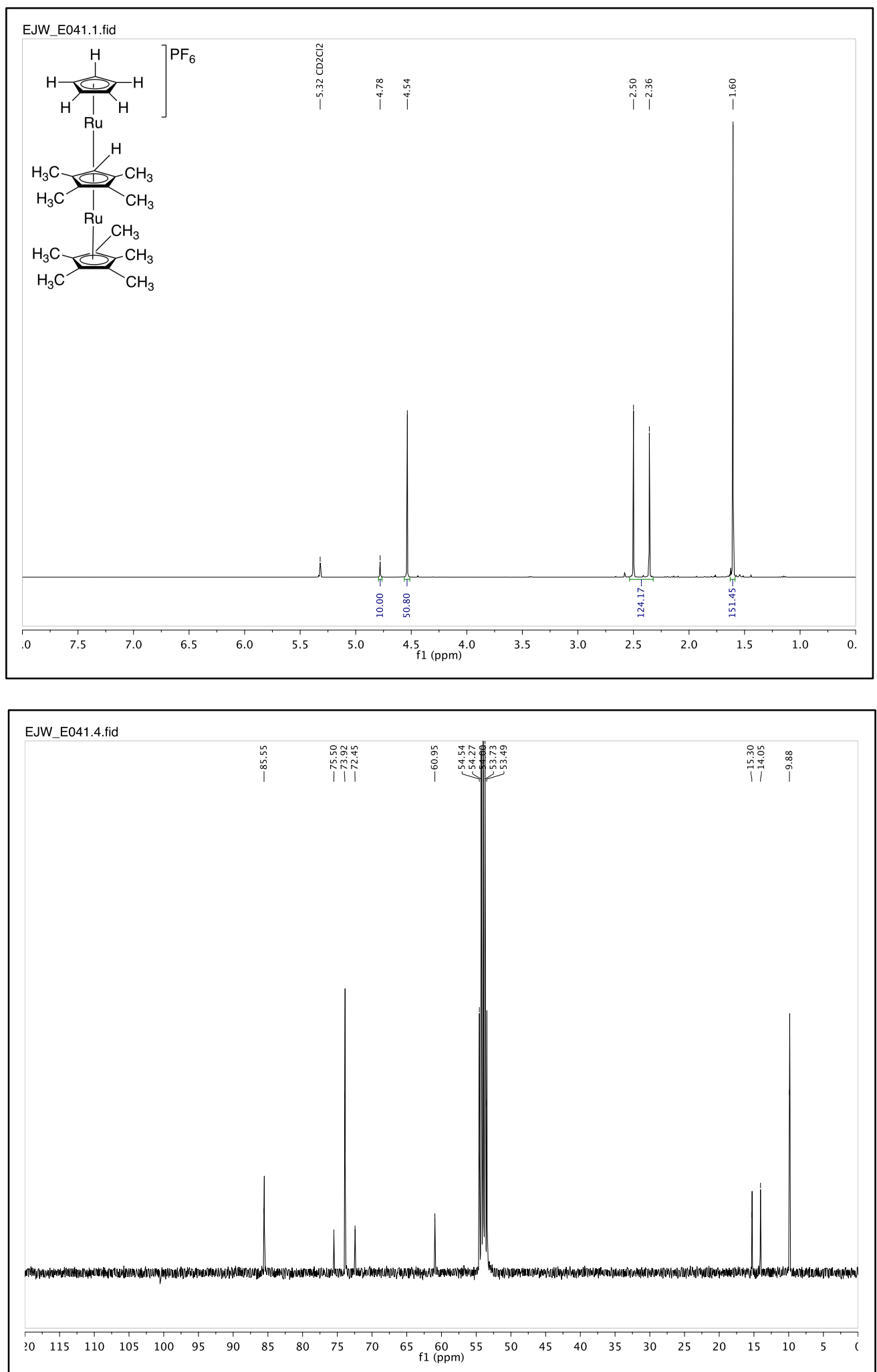
Figure S7. ${ }^{1} \mathrm{H},{ }^{13} \mathrm{C}\{\mathrm{H}\}$ NMR spectra for $\left[\mathrm{Cp} * \mathrm{Ru}\left(\mu-\mathrm{Cp}^{\prime}\right) \mathrm{RuCp}^{\prime}\right] \mathrm{PF}_{6}\left(\mathbf{5} \cdot \mathrm{PF}_{6}\right)$.
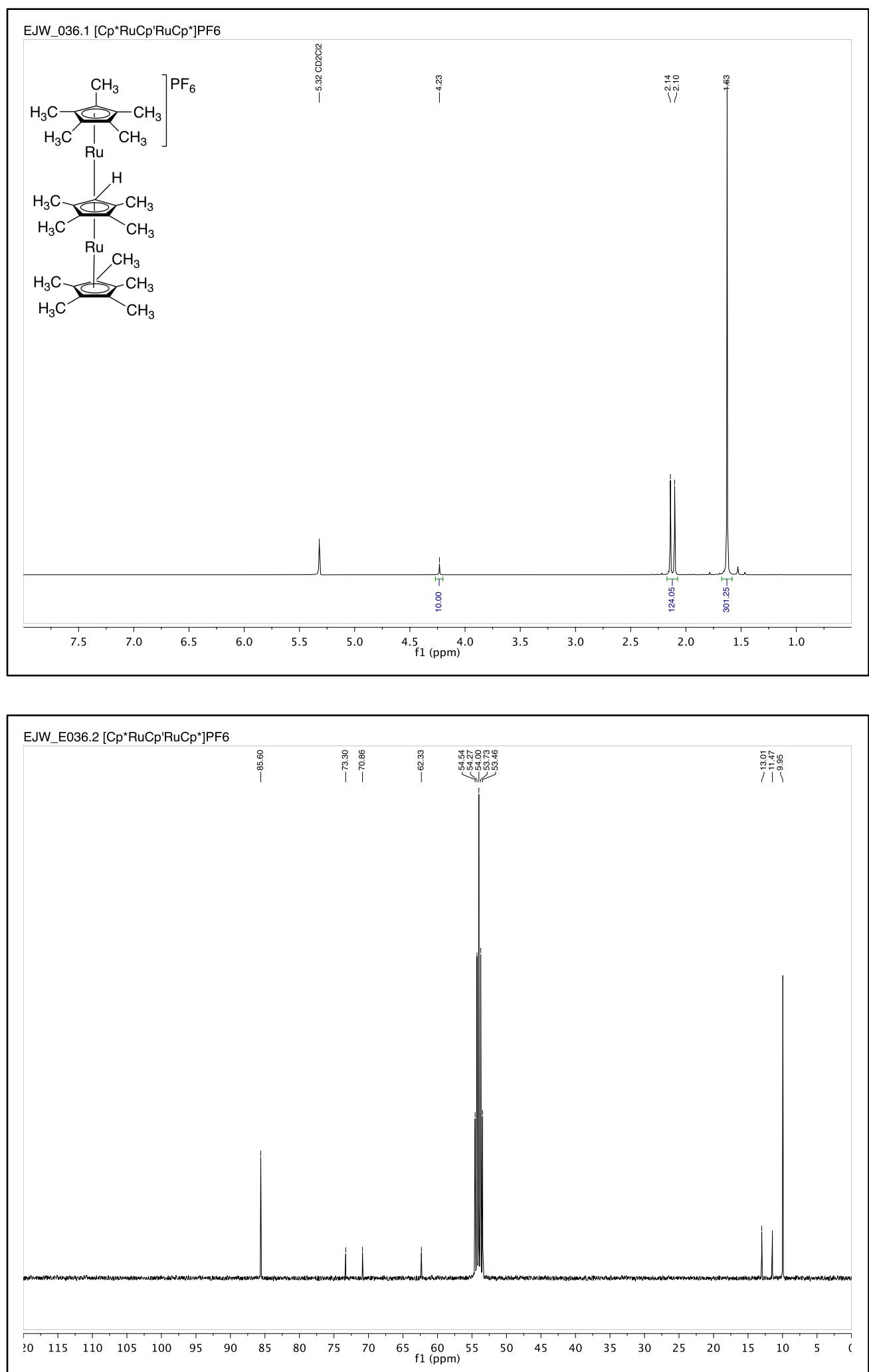
Figure S8. ${ }^{1} \mathrm{H},{ }^{13} \mathrm{C}\{\mathrm{H}\}$ NMR spectra for $\mathrm{Cp} * \mathrm{RuCp} \mathrm{p}^{\prime}(\mathbf{6})$.
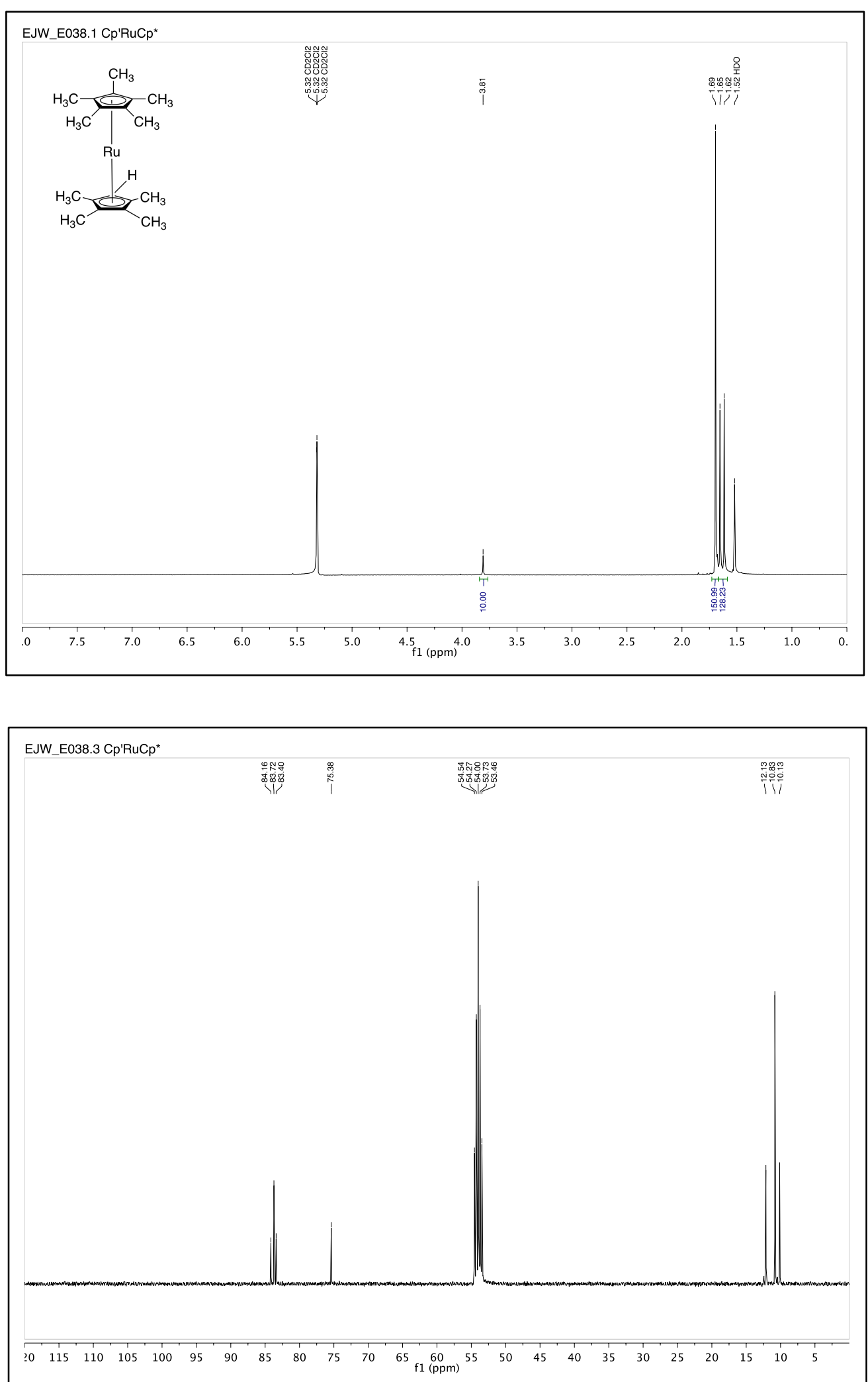